\title{
Un secolo di evoluzione del sistema ospedaliero
}

\author{
M. Rossetti
}

Da bambino e ginnasiale negli anni 30 a 40 volevo rimanere un montanaro ticinese, dove passavo come pastore, contadino, più tardi cacciatore romantico le mie vacanze più belle. La mia famiglia aveva programmato la medicina dicendomi: Biasca (la mia patria primaria) prepara un Ospedale, il patriziato ha già un fondo di 30000 franchi! La regione era intrisa di Ospedali: a $20 \mathrm{~km}$ il San Giovanni di Bellinzona, a $12 \mathrm{~km}$ Acquarossa, a Faido il Distrettuale e Santa Croce. Domando a mia madre un «colloquio di servizio»: Tu vuoi il medico, io il montanaro ... lasciami fare il veterinario di montagna ... La carriera andò diversamente.

Questa premessa personale per sottolineare come a quel tempo la rete ospedaliera era fitta e apprezzata dalla gente di ogni condizione, beninteso non solo nel Ticino. Si voleva l'ospedale possibilmente davanti agli occhi. La struttura tecnologica era minima in confronto ad oggi: il primario con un team di pochi collaboratori ruotanti era il perno, sorretto dall'esperienza, dal carisma, da una radiodiagnostica elementare (torace, transito baritato e clisma per l'internista, sistema osseo per il chirurgo - il radiologo specialista a turno giornaliero per tutta una regione era l'eccezione - la radioterapia era universitaria, nascente e temuta). Il laboratorio era semplice ma efficace per pochi esami di routine, al microscopio sedeva il primario e/o una laborantina.

«Di sacro non ha più che l'osso, di positivo solo il Wassermann!» per citare un esame allora automatico, senza chiedere al paziente, per escludere un'eventuale sifilide. Si pensi come reagirebbe il paziente oggi. Ho citato per ironia, un detto usato per distendere i nervi, in un'epoca in cui il team curante non parlava di ore di lavoro; si abitava nell'area ospedaliera e si accettavano la continuità come un privilegio professionale e le brevi vacanze quando possibile. Il personale infermieristico (ho vissuto con Suore religiose e professionisti laici d'ambo i sessi) concepiva l'azione e l'impatto come e più dei medici. Anche qui nessun problema d'orario e di continuità; le Suore religiose, per citare esempi indimenticabili, poche per molti pazienti, erano pronte giorno e notte, ritirate di tempo in tempo in cappella o nella Chiesa e vivevano pienamente per il compito, la pratica, l'ideale.
Consiglio la lettura del volume dell'amico e collega Urs Heim [1]: descrive con competenza e fascino aspetti e atmosfera della vita ospedaliera riassunta da me in queste poche righe. Quale evoluzione, da questi quadri dei decenni di allora fino ad oggi! L'Ospedale sulla porta di casa nei centri come nelle valli periferiche, con l'efficienza concentrata su persona ed esperienza di «generalisti» delle specialità principali (internista, chirurgo, ginecologo, pediatra) con piccolo team volonteroso, motivato, però ruotante a breve scadenza e l'infrastruttura tecnica sopra riassunta, scompare gradatamente per le ragioni di forza maggiore che commentiamo brevemente.

L'esplosione delle specialità, della tecnologia diagnostica e terapeutica e beninteso dei costi impongono concentrazioni geografiche, professionali e politiche che non toccano soltanto i grossi centri universitari e cantonali ma tutta la struttura nazionale ed internazionale.

Senza entrare in dettagli personali e tecnologici, il corpo medico e la popolazione devono accettare questi cambiamenti inesorabili che portano a vantaggi sensazionali nei risultati terapeutici, nel controllo di malattie, traumi e politraumi riducendo sempre più la mortalità, la morbidità e migliorando a media e lunga scadenza la qualità di vita. Quanto è andato perso nell'evoluzione-rivoluzione nel corso dell'ultimo mezzo secolo è in parte la componente umana come in tanti altri campi della nostra esistenza. Pazienti e famiglie devono oggi accettare spostamenti, trasporti, talora ritardi nelle date previste all'inizio, soggiorni ospedalieri sempre più brevi sotto pressione e beninteso i costi assicurati o no. Tutto ciò per ottenere risultati positivi impensabili nelle fasi passate, con questo compensando il rimpianto romantico dell'ospedale «famigliare» di allora a vista d'occhio.

Ciò che vogliamo per noi, per i nostri pazienti, per la formazione clinica, tecnica, etica, deontologica dei nostri giovani colleghi di oggi e di domani sono la volontà e la capacitä di quell'equilibrio talvolta difficile tra la supertecnologia e quella componente di «humanitas» sempre ancora essenziale nella nostra professione. Il rapporto morale tra il team curante e il paziente può diventare oggi tra le superstrutture, computer,
Prof. Dott. Mario Rossetti

Rennweg 51

CH-4052 Basilea 
diagrammi, documentazione rapida e continua e lo «stress» (un termine che ho sempre proibito di pronunciare nei miei gruppi e rapporti clinici) sovente difficile, il colloquio insufficiente sul piano umano.

Il paziente ha bisogno oggi più che mai, nelle fasi orientative e preparatorie, prima e dopo procedure diagnostiche e interventi terapeutici, della parola amica, tranquillizzante sopratutto davanti a problemi critici. Trasmettere insicurezza o addirittura terrore nelle fasi delicate del rapporto tra il medico e il paziente (statisticamente 3 mesi di vita!) è un psicotrauma tremendo in gran parte dei casi pur essendo sovente scientificamente oggettivo. Orientamenti e spiegazioni, delicate sopratutto quando sono coinvolti più colleghi - oggi la norma - influiscono non solo sulla psico-somatica, ma anche sulla resistenza organica, sul sistema immunitario e sulla qualità di vita che vogliamo sostenere in situazioni di ogni gravità. Questa è dimensione deontologica continua e primaria nostra, di noi tutti.

Così avviciniamo l'umanità del sistema ospedaliero di allora, con medici, personale e architettura «davanti agli occhi» di casa, con il sistema superefficace di oggi, in una fusione positiva di due realtà.

\section{Letteratura}

1 Heim UFA. Leben für andere. Die Krankenpflege der Diakonissen und Ordensschwestern in der Schweiz. Basel: Schwabe; 1998. 